\title{
Axial Ward Identity and the Schwinger Mechanism: Applications to the Real-Time Chiral Magnetic Effect and Condensates
}

\author{
Patrick Copinger, ${ }^{1}$ Kenji Fukushima, ${ }^{1}$ and Shi $\mathrm{Pu}^{1,2}$ \\ ${ }^{1}$ Department of Physics, The University of Tokyo, 7-3-1 Hongo, Bunkyo-ku, Tokyo 113-0033, Japan \\ ${ }^{2}$ Department of Modern Physics and Interdisciplinary Center for Theoretical Study, \\ University of Science and Technology of China, Hefei, Anhui 230026, China
}

(Received 26 July 2018; revised manuscript received 2 November 2018; published 28 December 2018)

\begin{abstract}
We elucidate chirality production under parity breaking constant electromagnetic fields, with which we clarify qualitative differences in and out of equilibrium. For a strong magnetic field the pair production from the Schwinger mechanism increments the chirality. The pair production rate is exponentially suppressed with mass according to the Schwinger formula, while the mass dependence of chirality production in the axial Ward identity appears in the pseudoscalar term only. We demonstrate that, in a realtime formulation with in and out states, the axial Ward identity with an in-in expectation value leads to a chirality production rate consistent with the Schwinger formula, while the axial anomaly with an in-out expectation value is canceled by the pseudoscalar condensate for any mass. We illuminate that such an inand out-state formulation clarifies subtleties in the chiral magnetic effect in and out of equilibrium, and we discuss further applications to real-time condensates.
\end{abstract}

DOI: 10.1103/PhysRevLett.121.261602

Introduction.-Chirality is a topical keyword for anomalous phenomena in physics and related subjects. In the context of high-energy physics in which the fermion mass is often neglected, the chirality and the helicity are identifiable, which has also motivated a modern redefinition of chirality in chemistry [1].

The most notable feature of chirality in relativistic fermionic systems is the realization of the quantum anomaly. Since relativistic fermionic dispersion relations are realized in not only 2D but also 3D materials, as in the Weyl and Dirac semimetals [2-5], it is of paramount importance to probe the chiral anomaly in laboratory experiments, with optical environments as well as in quantum chromodynamics (QCD). One proposed signature for the chiral anomaly is the negative magnetoresistance [6], which is a signal of chiral anomaly through the chiral magnetic effect [7]. For the first experimental detection based on simple theoretical arguments, see Ref. [8], and for the resummed fieldtheoretical calculation of the negative magnetoresistance, see Ref. [9].

In all ideas to access the chiral anomaly, the generation of finite chirality imbalance is indispensable. The simplest optical setup is, as discussed in Ref. [10], parallel electric and magnetic fields. Then, the chirality production rate is

Published by the American Physical Society under the terms of the Creative Commons Attribution 4.0 International license. Further distribution of this work must maintain attribution to the author(s) and the published article's title, journal citation, and DOI. Funded by SCOAP . related to the Schwinger mechanism as used in Refs. [10,11], and at the same time it is dictated by the axial Ward identity as argued in Ref. [12]. Such a simple electromagnetic configuration is also useful to test ideas in real-time numerical simulations $[13,14]$.

Even though the parallel electromagnetic fields are simple to treat, there are still some controversies especially on different manifestations of the chiral anomaly in and out of equilibrium. In this Letter we clarify these controversies by addressing the following two closely related problems, namely: (i) The effect of fermion mass $m$; it is quite often assumed that the mass dependent term can be dropped from the axial Ward identity if $m=0$, but this is not always justified. (ii) Real-time and Euclidean observables; the $m$ dependence is totally different depending on how to take the expectation value in the presence of electric fields.

Answering these questions will naturally lead us to a clear picture of chiral dynamics. Moreover, we will see that our present considerations have many applications to be studied in the future.

An enigma.-We choose constant and parallel electric $E$ and magnetic $B$ fields in the three-axis direction. Then, the celebrated formula for the Schwinger mechanism reads,

$$
\omega=\frac{e^{2} E B}{4 \pi^{2}} \operatorname{coth}\left(\frac{B}{E} \pi\right) \exp \left(-\frac{\pi m^{2}}{e E}\right)
$$

for the pair production rate (for a comprehensive review, see Ref. [15]). In a particular limit of strong $B$ (i.e., $\sqrt{e B}$ being the largest mass scale in a system), the spin direction is completely aligned along $B$, so that particles have 


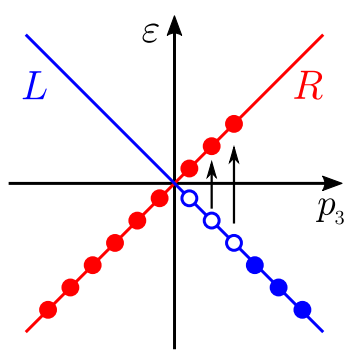

FIG. 1. Schematic picture of the pair production in a reduced $(1+1)$-dimensional system in the LLLA for $m=0$; right-handed particles and left-handed antiparticles and thus net chirality are generated with parallel $E$ along the three-axis direction.

definite chirality in a reduced $(1+1)$-dimensional system emerging in the lowest Landau level approximation (LLLA). The right-handed $(R)$ particles increase and the left-handed $(L)$ particles decrease creating $L$ antiparticles under $E$, as sketched in Fig. 1.

A pair of $R$ and $\bar{L}$ production thus changes the chirality by two, leading to a relation as used in Ref. [10],

$$
\omega \stackrel{B \gg E}{\longrightarrow} \frac{e^{2} E B}{4 \pi^{2}} \exp \left(-\frac{\pi m^{2}}{e E}\right)=\frac{1}{2} \partial_{t} n_{5},
$$

where $n_{5}$ is the chiral charge density, that is an expectation value of $j_{5}^{0}$.

The right-hand side $\partial_{t} n_{5}$ is dictated by the axial Ward identity, i.e.,

$$
\partial_{\mu} j_{5}^{\mu}=-\frac{e^{2}}{16 \pi^{2}} \epsilon^{\mu \nu \alpha \beta} F_{\mu \nu} F_{\alpha \beta}+2 m \bar{\psi} i \gamma_{5} \psi
$$

on the operator level, where $\epsilon^{\mu \nu \alpha \beta} F_{\mu \nu} F_{\alpha \beta}=-8 E B$ for parallel $E$ and $B$ in the present setup. After taking the expectation value with $\left\langle j_{5}^{i}\right\rangle=0$ presumed (which is checked by explicit calculations in the method discussed later), the chirality production should follow,

$$
\partial_{t} \bar{n}_{5}=\frac{e^{2} E B}{2 \pi^{2}}+2 m\left\langle\bar{\psi} i \gamma_{5} \psi\right\rangle
$$

(The reason for changing $n_{5}$ by $\bar{n}_{5}$ will be explained below.) One might have thought that Eqs. (2) and (4) are consistent if $m=0$, which is frequently assumed in the literature. The justification is not so trivial, however, even for the $m=0$ case. Because parallel $E$ and $B$ make a parity breaking combination, $\left\langle\bar{\psi} i \gamma_{5} \psi\right\rangle \propto E B$ is anticipated. In fact, for the pseudoscalar condensate, Schwinger performed the calculation using the proper-time method to discuss the interaction between the neutral meson and the proton, which results in [16]

$$
\bar{P}:=\left\langle\bar{\psi} i \gamma_{5} \psi\right\rangle=-\frac{e^{2} E B}{4 \pi^{2} m} .
$$

This makes $\partial_{t} \bar{n}_{5}=0$ for any $m$ including even $m=0$ limit. This apparent contradiction between $\omega$ and $\partial_{t} \bar{n}_{5}$ is quite often overlooked in a naive treatment of dropping $m\left\langle\bar{\psi} i \gamma_{5} \psi\right\rangle$ for $m=0$. As a matter of fact, it is well known that an $m=0$ Abelian gauge theory could be quite different from a theory in the $m \rightarrow 0$ limit, where in the former the electric charge is completely shielded [17]. In QCD, $m \approx 0$ only approximately holds, so we should consider the latter limit judiciously.

In state and out state.-To resolve this puzzle, the crucial observation is that the vacua at $t \rightarrow \pm \infty$ are not identical when an $E$ field is imposed, even if $E$ itself is static. If we take $A_{0}=0$ gauge, $A_{3}(t)$ is needed for $E$ along the threeaxis direction, and the in state $\mid$ in $\rangle$ and the out state $\mid$ out $\rangle$ are different by $A_{3}( \pm \infty)$, which are connected by the Bogoliubov transformation. Let us introduce the following notation for the in- and out-state expectation values:

$$
\langle\mathcal{O}\rangle:=\langle\operatorname{out}|\mathcal{O}(t)| \text { in }\rangle, \quad\langle\langle\mathcal{O}\rangle\rangle:=\langle\text { in }|\mathcal{O}(t)| \text { in }\rangle
$$

for an operator $\mathcal{O}(t)$ in the Heisenberg representation. In quantum field theory calculus the generating functional represents an amplitude $\langle$ out|in〉, and we must clearly distinguish,

$$
\bar{n}_{5}:=\left\langle j_{5}^{0}\right\rangle, \quad n_{5}:=\left\langle\left\langle j_{5}^{0}\right\rangle\right\rangle .
$$

In principle, one can utilize the Schwinger closed time path formalism to deal with $\langle\langle\mathcal{O}\rangle\rangle$. The straightforward approach is, however, technically complicated for our present problem, especially with $A_{3}(t)$.

Fortunately, for the constant $E$ case, a much simpler formulation has been known. According to Ref. [18] the two-point correlation function takes the following propertime representation,

$$
S_{\text {in }}^{c}(x, y):=i\langle\langle T \psi(x) \bar{\psi}(y)\rangle\rangle=\left(i D_{x}+m\right) \Delta_{\text {in }}^{c}(x, y),
$$

where we can express $\Delta_{\text {in }}^{c}(x, y)$ using $z=x-y$ and the proper time $s$ as

$\Delta_{\text {in }}^{c}(x, y)=\left(\theta\left(z_{3}\right) \int_{\Gamma^{>}} d s+\theta\left(-z_{3}\right) \int_{\Gamma^{<}} d s\right) g(x, y, s)$.

Here, the integration contours $\Gamma^{>}$and $\Gamma^{<}$are shown in Fig. 2, respectively, where we introduced a slightly different (but equivalent) representation from Ref. [18].

After some calculations we find the integration kernel with parallel $E$ and $B$ as

$$
\begin{aligned}
g(x, y, s)= & \frac{e^{2} E B}{(4 \pi)^{2}} \sinh ^{-1}(e E s) \sin ^{-1}(e B s) \\
& \times \exp \left[-i\left(\frac{1}{2} e F \sigma+m^{2}\right) s+i \varphi(x, y, s)\right],
\end{aligned}
$$

where $m^{2}$ should be understood as $m^{2}-i \epsilon$ for convergence in Minkowskian spacetime. We used a shorthand notation, $F \sigma=F_{\mu \nu} \sigma^{\mu \nu}$, whose explicit form is 


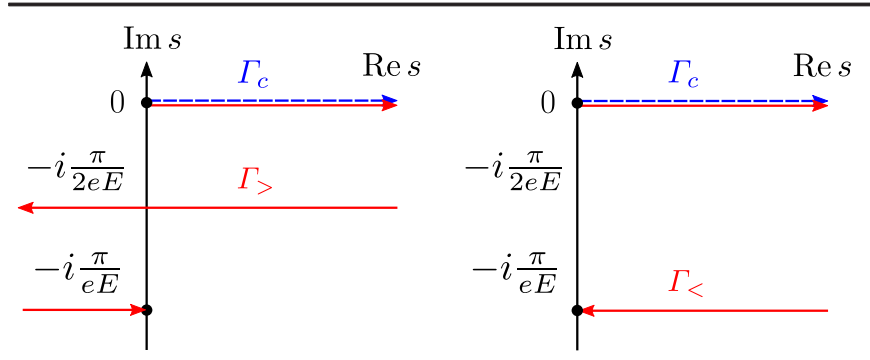

FIG. 2. For the standard propagator, $i\langle T \psi(x) \bar{\psi}(y)\rangle$, the propertime integration should go along $\Gamma_{c}$ (dashed line), while for $S_{\text {in }}^{c}$ the contour is complexified as $\Gamma_{>}$for $z_{3}>0$ and $\Gamma_{<}$for $z_{3}<0$.

$$
e^{-i(1 / 2) e F \sigma s}=\left(\begin{array}{ccc}
e^{-e(E-i B) s} & & 0 \\
& e^{e(E-i B) s} & \\
& & e^{e(E+i B) s} \\
0 & & e^{-e(E+i B) s}
\end{array}\right)
$$

in the chiral representation of $\gamma^{\mu}$. The coordinate dependent phase is

$$
\varphi(x, y, s)=\frac{1}{2} x e F y-\frac{1}{4} z \operatorname{coth}(e F s) e F z
$$

where $x e F y=x_{\mu} e F^{\mu \nu} y_{\nu}$, etc. Armed with the explicit form of the two-point correlation function, we are ready for concrete calculations.

An éclaircissement.—Let us first consider,

$$
P:=\left\langle\left\langle\bar{\psi} i \gamma_{5} \psi\right\rangle\right\rangle=-\lim _{y \rightarrow x} \operatorname{tr}\left[\gamma^{5} S_{\mathrm{in}}^{c}(x, y)\right]
$$

For this expectation value a term involving $D$ in Eq. (8) vanishes in the $y \rightarrow x$ limit, and so $P=$ $-i m \operatorname{tr}\left[\gamma^{5} \Delta_{\text {in }}^{c}(x, y \rightarrow x)\right]$, leading to

$P=-4 i \frac{m e^{2} E B}{(4 \pi)^{2}} \int_{\Gamma} d s e^{-i m^{2} s}=-\frac{e^{2} E B}{4 \pi^{2} m}\left(1-e^{-\pi m^{2} /(e E)}\right)$,

where $\Gamma$ is either $\Gamma^{>}$or $\Gamma^{<}$, which is irrelevant for the computation of $P$ since there is no pole in the integrand. Then, taking the $\langle\langle\ldots\rangle\rangle$ expectation value of Eq. (3) and plugging Eq. (14) into it, we see that Eq. (2) holds as it should. We note that Eq. (14) was conjectured in Ref. [11] from the Schwinger formula, but the clear recognition of differences with in and out states as revealed in this work was missing.

Interestingly, using our method, we can directly evaluate $n_{5}$ from Eq. (8) to reach a consistent conclusion, i.e., $n_{5}=$ $2 \omega t$ correctly (where $t$ appears from the phase space volume [18]), while we find $\bar{n}_{5}=0$ as is again consistent with $\partial_{t} \bar{n}_{5}=0$. Although the details for the $n_{5}$ and $\bar{n}_{5}$ calculations are extremely intriguing on their own, we will spell out stepby-step procedures in a follow-up publication.

Now, we have clarified that $n_{5}$ in the Schwinger mechanism should be a real-time observable as defined by $\langle\langle\mathcal{O}\rangle\rangle$ in Eq. (6), which has a demystified consistency with the axial Ward identity. Then, it is interesting to consider what $\partial_{t} \bar{n}_{5}=$ 0 means. To answer this question it would be convenient to Wick-rotate the time (not the proper time) as $t \rightarrow \tau$ to switch to Euclidean theory, in which $\mid$ in $\rangle$ and $\mid$ out $\rangle$ are the ground states at $\tau=0$ and $\tau=\beta$, respectively. Then, what we computed by $\langle\mathcal{O}\rangle$ corresponds to the static and Euclidean expectation value at $\beta=\infty$ (i.e., zero temperature).

The situation is even more transparent if we performed the Euclidean Monte Carlo calculation on the lattice. As discussed in Ref. [19], even with real $E$ (for which two flavors are oppositely charged to avoid the sign problem, which may well be called an "isospin" $E$ [19]), the lattice simulation always measures $\langle\mathcal{O}\rangle$ and cannot describe the real-time particle production; only the charge distribution deformed by $E$ or the polarization in the final state is observed.

We note that $\partial_{t} \bar{n}_{5}=0$ should be interpreted as $\left\langle\partial_{\tau} j_{5}^{\tau}\right\rangle=0$ with the Euclidean time derivative. One might be surprised by the fact that the anomaly effect completely vanishes with the Euclidean expectation value, but one can easily understand why it should be so for the $m=0$ case. The topological properties of the ground state are characterized by the $\theta$ angle, and the ground state energy is independent of $\theta$ if there is any $m=0$ fermion. Then, there is no nonzero topological charge and no chirality flip. In this way, $\left\langle\partial_{\tau} j_{5}^{\tau}\right\rangle=0$ is actually demanded for $m=0$ and the careful $m \rightarrow 0$ limit of the second term in Eq. (4), which may not always vanish, is crucial. See also Ref. [20] for an independent approach leading to the same conclusion as ours for $m=0$. We must emphasize that it is astonishing that $\left\langle\partial_{\tau} j_{5}^{\tau}\right\rangle=0$ holds for any $m$, about which we have no simple explanation.

One important extension along these lines of $n_{5}$ is found in the calculation of the chirality fluctuation. In particular, in the high-energy nuclear collision experiments, fluctuations averaged over many collision events or even with a single event are important physical observables. Then, it is an urgent task in theory to compute, $\chi_{5}:=\left(\left\langle\left\langle N_{5}^{2}\right\rangle\right\rangle-\left\langle\left\langle N_{5}\right\rangle\right\rangle^{2}\right) / V$, where $N_{5}:=\int d^{3} x j_{5}^{0}(x)$ to quantify the idea of the local parity violation. Our setup with parallel $E$ and $B$ is very simple but mimics the initial condition of the nuclear collision known as the Glasma flux tubes. Again, if we performed the lattice Monte Carlo simulation, we would get $\bar{\chi}_{5}:=\left(\left\langle N_{5}^{2}\right\rangle-\left\langle N_{5}\right\rangle^{2}\right) / V$, which is qualitatively different from our interested $\chi_{5}$. As we already saw, the disconnected piece, $\left\langle\left\langle N_{5}\right\rangle\right\rangle=\int d^{3} x 2 \omega t$, grows linearly with time, and the term $\propto t^{2}$ in $\left\langle\left\langle N_{5}^{2}\right\rangle\right\rangle$ is subtracted in $\chi_{5}$. This also implies that a term $\propto t$ still remains in $\chi_{5}$, which is absent in $\bar{\chi}_{5}$. We actually find such a term $\propto t E B e^{-2 \pi m^{2} /(e E)}$, all the details about which shall be reported in a follow-up publication. Instead, below, we shall focus on local observables involving only one 
$S_{\text {in }}^{c}(x, y)$, namely, the expectation values of the current and the scalar operators.

Chiral magnetic effect in and out of equilibrium.-It is a straightforward exercise to compute the vector current associated with the chiral magnetic effect. In Ref. [10] the answer was inferred from the Lorentz transformation of the Schwinger formula. With our method, although it is a tedious calculation, particularly complex poles in the proper-time integration need careful treatments, after all we arrive at

$$
j^{3}=\left\langle\left\langle\bar{\psi} \gamma^{3} \psi\right\rangle\right\rangle=-\lim _{y \rightarrow x} \operatorname{tr}\left[\gamma^{3} S_{\mathrm{in}}^{c}(x, y)\right]=2 \omega t
$$

which is the right answer for strong $B$; generated $n_{5}$ is immediately converted to $j^{3}$. We note that the above result does not rely on the LLLA; we simply used the LLLA argument to relate the axial Ward identity and the Schwinger formula, but our results of $P, n_{5}, j^{3}$ are all valid beyond the LLLA.

Here, interestingly, a simple calculation yields

$$
\bar{j}^{3}:=\left\langle\bar{\psi} \gamma^{3} \psi\right\rangle=0
$$

in the same way to obtain $\bar{n}_{5}=0$. This is a very important result to demonstrate unambiguously that the chiral magnetic effect does not exist in Euclidean spacetime. In short, in equilibrium, there is no chirality generation, and there is no topological current.

Such a statement about equilibrium chiral magnetic effect itself is not quite new, see Ref. [21]. Some confusion is attributed to a chiral chemical potential $\mu_{5}$; with $\mu_{5}$ it seems that the equilibrium chiral magnetic effect is always exist [7] but one should be aware of the fact that the introduction of $\mu_{5}$ implicitly assumes a system out of equilibrium (otherwise, $\mu_{5}$ should be vanishing). Thus, $\mu_{5}$ is a very useful bookkeeping device to access the correct physics out of equilibrium even within equilibrium framework. However, the price to pay is that qualitative differences in the chiral magnetic effect in and out of equilibrium cannot be clear as long as $\mu_{5}$ is incorporated. Equations (15) and (16) clearly show that the chiral magnetic effect is an intrinsically real-time phenomenon. Also we note that, if one performed the lattice Monte Carlo simulation with Euclidean or isospin real $E$ and $B$ trying to quantify a chiral magnetic current on the lattice, Eq. (16) predicts that the lattice answer for the current should be zero.

Dynamical chiral condensate.-Now, let us turn to another problem, that is, a scalar condensate (which is commonly called the chiral condensate in QCD). Since we already discussed the pseudoscalar condensates, $\bar{P}$ and $P$, it would be a quite natural extension of the study. The scalar condensate is as important as $\bar{P}$ and $P$ since it would induce constituent masses. In the absence of $E$, it is established that strong $B$ and a tiny interaction would inevitably lead to a finite chiral condensate, and this is referred to as the magnetic catalysis $[22,23]$. In this case the induced chiral condensate (apart from interaction contributions) reads,

$$
\begin{aligned}
\left.\bar{\Sigma}\right|_{E=0}:=\langle\bar{\psi} \psi\rangle_{E=0} & =-\frac{e B}{4 \pi^{2}} m \int_{\Gamma_{c}^{\Lambda}} \frac{d s}{s} e^{-i m^{2} s} \cot (e B s) \\
& \simeq-\frac{e B}{4 \pi^{2}} m \Gamma\left[0, m^{2} / \Lambda^{2}\right],
\end{aligned}
$$

where we can imaginary rotate [24] the proper time $i s \rightarrow s$ to make the above look identical to the expression in Refs. [22,23]. We can also approximate the incomplete gamma function as $\Gamma[0, x] \sim-\gamma_{E}-\ln x$ for small $x$, with $\gamma_{E}$ being the Euler-Mascheroni constant. The ultraviolet divergence is regularized by a shift of $\Gamma_{c}$ near the origin as $s=0 \rightarrow 1 / \Lambda^{2}$, which is denoted by $\Gamma_{c}^{\Lambda}$. From the first to the second line, $\operatorname{coth}(e B s) \sim 1$, corresponding to the LLLA, is used. The logarithmic singularity with respect to $m^{2}$ above is translated into a term $\sim m^{2} \ln \left(\Lambda^{2} / m^{2}\right)$ in the effective potential, which indicates a negative infinite curvature near $m \sim 0$, and this gives rise to the magnetic catalysis. In our present formulation it is easy to include a finite $E$;

$$
\begin{aligned}
\bar{\Sigma}= & -\frac{e^{2} E B}{4 \pi^{2}} m \int_{\Gamma_{c}^{\Lambda}} d s e^{-i m^{2} s} \cot (e B s) \operatorname{coth}(e E s) \\
& \simeq-\frac{e B}{4 \pi^{2}} m\left[\ln \frac{\Lambda^{2} e^{-\gamma_{E}}}{2 e E}-\operatorname{Re} \psi\left(\frac{i m^{2}}{2 e E}\right)-\frac{i \pi}{e^{\pi m^{2} /(e E)}-1}\right] .
\end{aligned}
$$

Here, we used approximations, $\operatorname{coth}(e B s) \sim 1$ and $e^{-m^{2} / \Lambda^{2}} \sim 1$, and wrote only terms nonvanishing in the large- $\Lambda^{2}$ limit. In the second line $\psi(x)$ represents the digamma function. Using an asymptotic expansion, $\psi(x) \sim$ $\ln x-1 / 2 x$ for large $x$, we can exactly recover Eq. (17) from the $e E \rightarrow 0$ limit of Eq. (18).

From Eq. (18) we see that the magnetic catalysis is overridden by $e E$ and there is no longer a logarithmic singularity with respect to $m^{2}$ (for related work on the phase structure with parallel $E$ and $B$, see Ref. [25]). Another noticeable feature of Eq. (18) is that the scalar condensate takes a complex value, which is analogous to a complex chiral condensate at finite strong- $\theta$ angle $[26,27]$. Since $\theta$ and $\boldsymbol{E} \cdot \boldsymbol{B}$ share the same quantum number, we can naturally anticipate the same behavior on the scalar condensate. An interesting point in Eq. (18) is that $\operatorname{Im} \bar{\Sigma}$ takes a form of the Fermi-Dirac distribution function with the energy over the temperature replaced by $\pi m^{2} /(e E)$.

In view of the fact that $P$ turns out to be quite different from $\bar{P}$ as in Eqs. (5) and (14), we may well anticipate such changes for the Euclidean and the real-time chiral condensates. In the same way as $P$, we can perform the calculation using $S_{\text {in }}^{c}(x, y)$, and then the imaginary rotated $(i s \rightarrow s$ ) expression takes the following form, 


$$
\begin{aligned}
\Sigma:=\langle\langle\bar{\psi} \psi\rangle\rangle= & -m \operatorname{tr}\left[\Delta_{\text {in }}^{c}(x, y \rightarrow x)\right] \\
= & -\frac{e^{2} E B}{4 \pi^{2}} m \int_{1 / \Lambda^{2}}^{\pi / e E-1 / \Lambda^{2}} d s e^{-m^{2} s} \\
& \times \operatorname{coth}(e B s) \cot (e E s) \\
\simeq & {\left[1-e^{-\pi m^{2} /(e E)}\right] \operatorname{Re} \bar{\Sigma}, }
\end{aligned}
$$

where we dropped a term involving $\not$ in the $y \rightarrow x$ limit, and kept only terms nonvanishing in the large- $\Lambda^{2}$ limit. It should be noted that the ultraviolet divergences appear from both edges of the integration range, that is, the integration without the cutoff would diverge in the ultraviolet or short-range limit of $z=x-y \rightarrow 0$ in exactly the same way near $s=0$ and $\pi /(e E)$ (in the LLLA). We thus regularized the integration by shifting both edges equally by $1 / \Lambda^{2}$. Then, this overall factor in Eq. (19) happens to be the same as that between $P$ and $\bar{P}$. We remark that no imaginary part appears in this case due to deformation of the integration contour, which is guaranteed by Hermiticity of physical operators.

From the point of view of the spontaneous symmetry breaking, Eq. (19) means that the generation of the chiral condensate and thus the constituent mass is more diminished as compared to the equilibrium case. From the dynamical point of view, Eq. (19) is a further significant result. It is sometimes a puzzling question whether the Schwinger mechanism produces the bare particles or the dressed particles. In other words, the question is whether the Schwinger critical mass is characterized by the bare mass or the constituent mass. Equation (19) indicates that, once the pair production is activated with $e^{-\pi m^{2} /(e E)} \sim 1$, the dynamical chiral condensate $\Sigma$ should melt, so that there is no constituent mass (which is defined by a mean-field approximation for the in-in expectation values). Our speculation could be tested experimentally with the Landau-Zener effect for interacting systems, i.e., breakdown of insulator into conductor which occurs through the Schwinger mechanism.

Summary.-We have clarified important differences associated with the in and out states in the presence of electric field $E$. In particular the mass dependence appears quite different, which resolves some controversies in the interpretations of the axial Ward identity, the chiral magnetic effect, and the chiral condensate. Here, to make our point not blurred by technicalities, we limited ourselves to the calculations involving one propagator only, namely, the expectation values of the current and the scalar operators. We will elsewhere discuss systematic computations of higher order observables, such as the real-time chirality fluctuations.

The authors thank Stefan Flörchinger, Xu-Guang Huang, Niklas Mueller, and Naoto Tanji for useful comments and discussions. K. F. is grateful for a warm hospitality at the Fudan University where he stays as a Fudan University Fellow. This work was supported by Japan Society for the Promotion of Science (JSPS) KAKENHI Grant No. 18H01211. S. P. was supported by the JSPS postdoctoral fellowship for foreign researchers.
[1] L. Barron, Chem. Phys. Lett. 123, 423 (1986).

[2] Z. Wang, Y. Sun, X.-Q. Chen, C. Franchini, G. Xu, H. Weng, X. Dai, and Z. Fang, Phys. Rev. B 85, 195320 (2012).

[3] Z. Wang, H. Weng, Q. Wu, X. Dai, and Z. Fang, Phys. Rev. B 88, 125427 (2013).

[4] M. Neupane, S.-Y. Xu, R. Sankar, N. Alidoust, G. Bian, C. Liu, I. Belopolski, T.-R. Chang, H.-T. Jeng, H. Lin, A. Bansil, F. Chou, and M. Z. Hasan, Nat. Commun. 5, 3786 (2014).

[5] Z. K. Liu, J. Jiang, B. Zhou, Z. J. Wang, Y. Zhang, H. M. Weng, D. Prabhakaran, S.-K. Mo, H. Peng, P. Dudin, T. Kim, M. Hoesch, Z. Fang, X. Dai, Z. X. Shen, D. L. Feng, Z. Hussain, and Y. L. Chen, Nat. Mater. 13, 677 (2014).

[6] D. T. Son and B. Z. Spivak, Phys. Rev. B 88, 104412 (2013).

[7] K. Fukushima, D. E. Kharzeev, and H. J. Warringa, Phys. Rev. D 78, 074033 (2008).

[8] Q. Li, D. E. Kharzeev, C. Zhang, Y. Huang, I. Pletikosic, A. V. Fedorov, R. D. Zhong, J. A. Schneeloch, G. D. Gu, and T. Valla, Nat. Phys. 12, 550 (2016).

[9] K. Fukushima and Y. Hidaka, Phys. Rev. Lett. 120, 162301 (2018).

[10] K. Fukushima, D. E. Kharzeev, and H. J. Warringa, Phys. Rev. Lett. 104, 212001 (2010).

[11] H. J. Warringa, Phys. Rev. D 86, 085029 (2012).

[12] A. Iwazaki, Phys. Rev. C 80, 052202 (2009).

[13] K. Fukushima, Phys. Rev. D 92, 054009 (2015).

[14] N. Müller, S. Schlichting, and S. Sharma, Phys. Rev. Lett. 117, 142301 (2016).

[15] G. V. Dunne, in From Fields to Strings: Circumnavigating Theoretical Physics, Ian Kogan Memorial Collection (3 volume set), edited by M. Shifman, A. Vainshtein, and J. Wheater (World Scientific, Singapore, 2004), pp. $445-522$.

[16] J. S. Schwinger, Phys. Rev. 82, 664 (1951).

[17] P. I. Fomin, V. A. Miransky, and Yu. A. Sitenko, Phys. Lett. 64B, 444 (1976).

[18] E. Fradkin, D. Guitman, and S. Shvartsman, Quantum Electrodynamics: With Unstable Vacuum, Springer Series in Nuclear and Particle Physics (Springer-Verlag, Berlin, 1991).

[19] A. Yamamoto, Phys. Rev. Lett. 110, 112001 (2013).

[20] M. Dvornikov and V. B. Semikoz, J. Cosmol. Astropart. Phys. 08 (2018) 021.

[21] N. Yamamoto, Phys. Rev. D 92, 085011 (2015).

[22] V. P. Gusynin, V. A. Miransky, and I. A. Shovkovy, Phys. Rev. Lett. 73, 3499 (1994); 76, 1005(E) (1996).

[23] V. P. Gusynin, V. A. Miransky, and I. A. Shovkovy, Phys. Lett. B 349, 477 (1995).

[24] To justify the imaginary rotation with singularities from $\operatorname{coth}(e B s)$ we should consider a nonzero $z_{1}=x_{1}-y_{1}$ and $z_{2}=x_{2}-y_{2}$ first, for which contours near and below these poles can be added without changing the integral. Then the imaginary rotation does not hit any poles, and finally $z_{1}$, $z_{2} \rightarrow 0$ can be taken.

[25] L. Wang, G. Cao, X.-G. Huang, and P. Zhuang, Phys. Lett. B 780, 273 (2018).

[26] D. Boer and J. K. Boomsma, Phys. Rev. D 78, 054027 (2008).

[27] K. Mameda, Nucl. Phys. B889, 712 (2014). 